\title{
Integratives und funktionelles Training zur Sturzprävention: Agility für Senioren?
}

\author{
Mareike Morat, Eric Lichtenstein, Oliver Faude und Lars Donath
}

\begin{abstract}
Beim Thema „Sturzprävention“ denken viele an alte Menschen, die in Gruppen oder als Einzelne unter Anleitung eines Therapeuten verhältnismäßig langweilige Übungen für das Gleichgewicht machen: Einbein- und Tandemstand, Seiltänzergang oder was auch immer für geeignet befunden wird, die Balance zu verbessern. Dass Sturzprävention aber auch mehr sein kann, zeigen unsere Autoren in diesem Beitrag über die Vorzüge Agility-basierter Trainingsansätze.
\end{abstract}

\section{Einleitung}

Senioren im Alter von über 60 Jahren werden bis zum Ende des 21. Jahrhunderts etwa $30 \%$ der Bevölkerung in westlichen Gesellschaften ausmachen[1]. Jedes Jahr fällt von diesen Senioren rund ein Drittel hin, und wiederum die Hälfte davon erleidet einen erneuten Sturz im folgenden Jahr [2]. Dabei gelten Stürze als „unerwartete Ereignisse, bei denen der Teilnehmer unbeabsichtigt auf dem Boden [...] zur Ruhe kommt“ [3]. Verletzungen, die dabei entstehen, sind die Hauptursache für Krankenhausaufenthalte und führen zu steigenden direkten und indirekten Kosten im Gesundheitswesen [4]. Die Zahl der sturzbedingten Verletzungen steigt mit dem Alter an, und sie tragen wesentlich zum früheren Auftreten von alltagsbedingten Einschränkungen bei [5].

Gleichgewichts- und Krafttraining kann neuromuskuläre Sturzrisikofaktoren (Surrogatparameter) und Sturzraten verbessern [6]. Neben konventionellem Kraft- und Gleichgewichtstraining werden auch exzentrische Übungen [7], Übungen zur Steigerung der Maximal- und Schnellkraft [8], Übungen für Rumpfkraft und -kontrolle [9][10], sowie statisches, dynamisches und perturbiertes (mit Störeinflüssen durchgeführtes) Gleichgewichtstraining empfohlen [11]. Auch eine gut ausgeprägte Ausdauerleistung kann möglicherweise Ermüdungserscheinungen der posturalen Kontrolle bei sportbezogenen Aktivitäten oder Aktivitäten des täglichen Lebens verringern [12].

In der Vergangenheit wurden zahlreiche Sturzrisikofaktoren, wie z. B. asymmetrische und abnehmende Explosivkraft der unteren Extremitäten [13] oder beeinträchtigte posturale Kontrolle, [8] identifiziert. Es wurde außerdem gezeigt, dass regelmäßiges Kraft- und Gleichgewichtstraining das Potenzial hat, altersbedingte Degenerationen zu verzögern [8]. So können Senioren mit gut entwickelter Kraftfähigkeit der unteren Extremitäten (speziell der Plantarflexoren) und mit adäquater posturaler Kontrolle ihr Sturzrisiko um bis zu 50 \% reduzieren [14]. Entsprechende Trainingsprogramme sollten vor allem regelmäßig durchgeführt, vielseitig und für Senioren ansprechend gestaltet werden [15].

Basierend auf diesen Forschungsergebnissen existieren vielversprechende „Best Practice“-Trainingsempfehlungen [16]. Physische Leistungsindikatoren wie Balance, Kraft und Ausdauer werden in Trainingsrichtlinien in der Regel jedoch unabhängig voneinander empfohlen, was ein recht zeitaufwendiges Training zur Folge hat. Deshalb haben wir einen funktionellen, Agility-basierten Trainingsansatz entwickelt, dessen Ziel es ist, Aspekte der Wahrnehmung und Entscheidungsfindung (z. B. visuelle Wahrnehmung, situative räumliche Orientierung, Erkennen und Antizipieren von Bewegungsmustern), Richtungsänderungen (einschließlich reaktiver Bewegungen und schneller konzentrischer sowie exzentrischer Muskelkontraktionen) und kardiovaskuläres Training zu kombinieren und dabei die Intensität, Komplexität und kognitive Herausforderung individuell anzupassen [17].

\section{Trainingsbasierte Sturzprävention: verfügbare „Best Practice“-Richtlinien}

Tägliche moderate bis starke körperliche Aktivität gilt als primärpräventive Strategie zur Vermeidung von Stür- 
zen, wobei das reine „Aktivsein“ nicht als ausreichende Maßnahme angesehen werden kann [18]. Das American College of Sports Medicine (ACSM) und die American Heart Association (AHA) empfahlen im Jahr 2007 selbstständigen gefährdeten Senioren, Gleichgewichtsübungen durchzuführen, um das Sturzrisiko zu senken [19]. Diese Empfehlungen wurden zwei Jahre später von ChodzkoZajko et al. (2009) präzisiert. Sie fügten hinzu, dass ein multimodaler Ansatz, der Kraft- und Gleichgewichtstraining sowie Tai Chi umfasst, das Sturzrisiko bei gefährdeten Senioren bedeutsam senken kann [9]. Jedoch basiert die Mehrzahl dieser Befunde auf kleinen Fallzahlen oder Observierungsstudien bzw. unkontrollierten oder nicht randomisierten Studien.

In einer Cochrane-Metaanalyse von 2012 wurden Sturzraten und das Sturzrisiko aus 159 präventiven Interventionsstudien mit einer Gesamtheit von fast 80000 Probanden zusammengefasst [14]. Die Analyse ergab, dass ein multimodales Training das Sturzrisiko um durchschnittlich 15\% reduzierte, unabhängig davon, ob es als Heim- oder Gruppentraining organisiert war. Diese Ergebnisse wurden in einer Metaanalyse von Sherrington et al. (2017) ergänzt und in Bezug auf die Trainingsmodalität spezifiziert: 88 randomisierte, kontrollierte Studien (RCTs) ergaben eine generelle Reduktion der Sturzrate um 21 \% [6]. Die Effekte waren am größten, wenn Balancetraining involviert war und insgesamt für mehr als drei Stunden pro Woche trainiert wurde (39\% Reduktion der Sturzrate) [6]. Eine MetaRegression konnte zeigen, dass die Reduktion der Sturzrate am größten war, wenn das Gehen ausgeschlossen und anspruchsvolles und regelmäßiges Gleichgewichtstraining im Umfang von zweimal einer Stunde pro Woche integriert wurde [20]. Eine Abnahme der Sturzrate um 42\% wurde in einer restriktiveren Metaanalyse mit 44 Studien festgestellt, die

- stark herausfordernde Gleichgewichtsübungen,

- eine hohe Dosierung und

- kein Gehen

beinhalteten [20]. Neuste Ergebnisse der überarbeiteten Metaanalyse von Sherrington et al. (2017) ergaben jedoch, dass Gangtraining in Anbetracht der Beliebtheit, gewisser gesundheitsfördernder Effekte und der erfolgreichen Durchführung in einigen Untersuchungen mit Vorsicht durchaus als ergänzendes Training eingesetzt werden kann [6].

\section{Übersichtsarbeiten weisen vor allem auf ein erforderliches Trainings- volumen von mindestens 3 Stunden pro Woche und auf die Bedeutung von herausforderndem Balance- training zum Zwecke der Sturz- prävention hin.}

Balancetraining sollte unter Berücksichtigung dieser Ergebnisse folgenden „Best practice“-Empfehlungen entsprechen: Übungen sollten

- Standübungen mit graduell instabiler werdender Unterstützungsfläche umfassen (z. B. Zweibeinstand, Semi-Tandemstand, Tandemstand, Einbeinstand),

- dynamische und reaktive Bewegungen integrieren, bei denen der Körperschwerpunkt aus der Ruhe gebracht wird (z. B. Tandemgang, Drehungen),

- postural relevante Muskelgruppen beanspruchen (z. B. Fersenstand, Zehenstand) und

- sensorischen Input variieren (z. B. Stehen mit geschlossenen Augen) [9][10].

Obwohl dies weniger ausführlich untersucht wurde, erscheint es nützlich, Gang- und Balancetraining mit zusätzlichen kognitiven oder motorischen Aufgaben zu kombinieren, da das statische und dynamische Gleichgewicht von Senioren durch Störeinflüsse negativ beeinflusst werden kann [11]. Wenn kognitive Ressourcen zur Bewältigung von Dual- oder Multi-Task-Aufgaben genutzt werden, scheint dies auf Kosten der posturalen Kontrolle zu geschehen, wodurch ein höheres Sturzrisiko in Situationen, in denen die Aufmerksamkeit auf verschiedene Aufgaben verteilt werden muss, zu begründen ist [21]. Dualoder Multi-Task-Aufgaben sind sowohl für Trainings- als auch für Testzwecke geeignet. Ein Rahmenplan für gleichgewichtsbezogene Trainingsschwerpunkte ist in $\mathbf{A b b} \mathbf{1}$ systematisiert worden.

In Ergänzung zu diesen Empfehlungen für Gleichgewichtstraining existieren ebenfalls Empfehlungen für das Krafttraining [8]. Meist wurde in Untersuchungen Krafttraining bei Senioren entweder im hochintensiven Bereich mit moderater Bewegungsgeschwindigkeit (mit 70-89\% des Einwiederholungsmaximums $=1 \mathrm{WM}$ ) oder mit maximalen Geschwindigkeiten in der konzentrischen Phase bei $20-80 \%$ des 1 WM (Schnellkrafttraining) durchgeführt (siehe Review von Granacher et al. [8]). Auch Programme mit „negativem“ Krafttraining, welche exzentrische Übungen in den Vordergrund stellen, wurden angesichts ihrer Besonderheit, hohe mechanische Belastung bei niedrigem Energieaufwand zu erzeugen, erfolgreich umgesetzt [7]. Bei dieser Trainingsform ist jedoch Vorsicht geboten (z. B. qualifiziertes Personal, konzentrierte Unterstützung, spezielle Geräte), um die Verletzungsgefahr zu minimieren (korrekte Bewegungen, Vermeidung von Scherkräften).

Aus den Ergebnissen verfügbarer RCTs zu traditionellem Krafttraining lassen sich folgende Trainingsempfehlungen formulieren: Wenn Krafttraining mindestens zweimal pro Woche durchgeführt wird, kann es bemerkenswerte Verbesserungen der Muskelhypertrophie, der Kraft und der Leistung zur Folge haben: Für das Training sollten eine moderate bis hohe Intensität (60-80\% des 1 WM) und ein moderates Volumen (zwei bis drei Sätze pro Übung) geplant sein [22]. Dabei sollten sechs bis acht Übungen insgesamt 


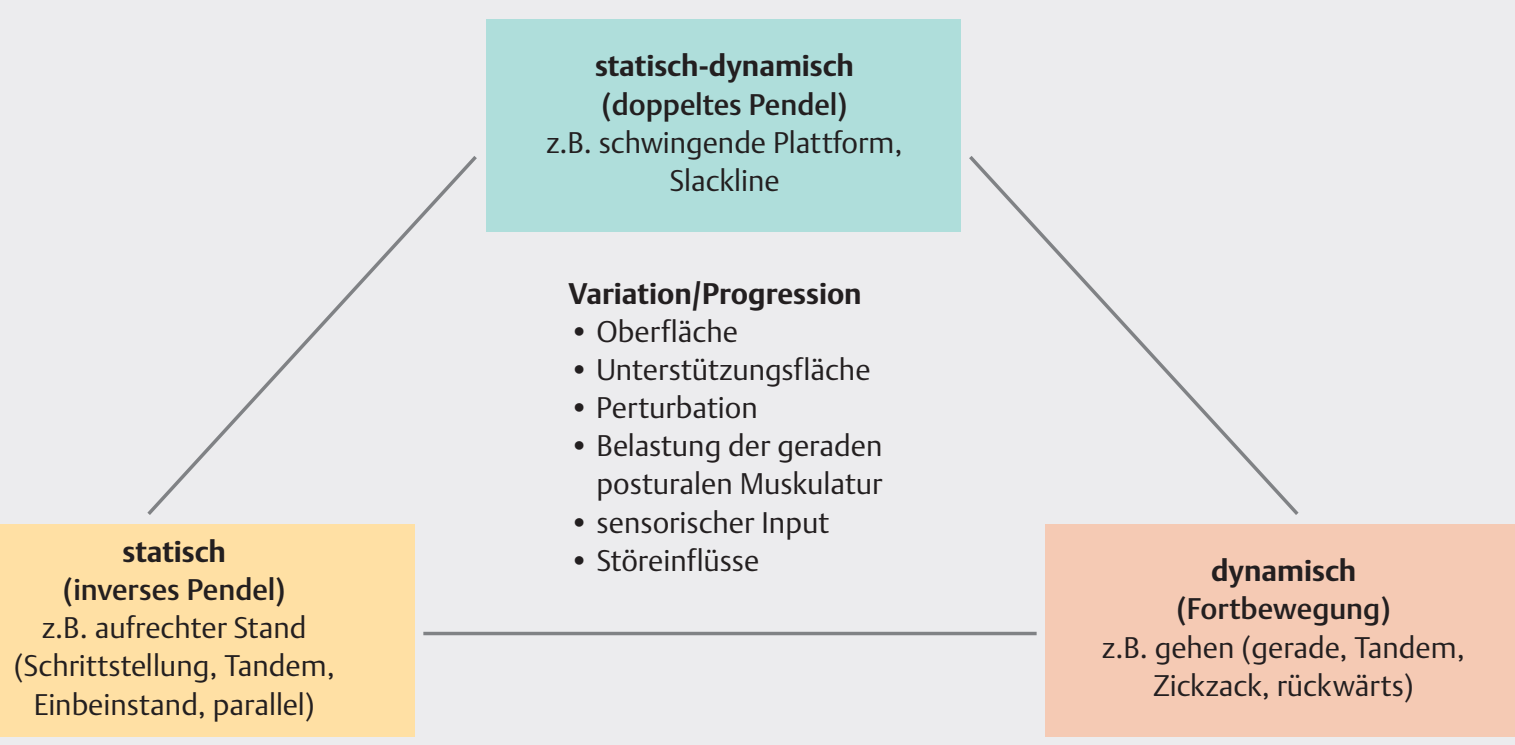

Abb. 1 Aspekte und Prinzipien des Gleichgewichtstrainings [8][10][11][16]: Differenzierung des Gleichgewichts in dynamisches, statisches und statisch-dynamisches Gleichgewicht und Möglichkeiten der Variation/Progression von Gleichgewichtsübungen (Quelle: Thieme Gruppe).

und acht bis zwölf Wiederholungen pro Satz durchgeführt werden. Transfereffekte eines solchen Krafttrainings auf die Balancefähigkeit scheinen limitiert zu sein. Trotzdem können Senioren mit höherer Kraftfähigkeit nach Perturbationen (externen Störeinflüssen) eher das Gleichgewicht wiedererlangen [23]. Dementsprechend reduzieren traditionelle Krafttrainingsprogramme das Sturzrisiko und können die Folgen von Stürzen minimieren [23]. Folglich sollte traditionelles Krafttraining zwar nicht die einzige Form von Training sein, es sollte aber in multifaktorielle Ansätze neuromuskulären Trainings integriert werden [8].

\section{Krafttraining reduziert das \\ Sturzrisiko und die Folgen von \\ Stürzen und sollte daher immer \\ in ein vielseitiges Training zur \\ Sturzprävention integriert werden.}

Darüber hinaus ist eine schnelle Kraftentfaltung (Explosivkraft) von besonderer Bedeutung, da sie in Situationen, in denen das Gleichgewicht bedroht ist, besonders relevant zu sein scheint [24]. Da die Explosivkraft in traditionellen Krafttrainingsprogrammen nicht ausreichend trainiert wird, wurden Trainingsprogramme zur Steigerung der schnellen Kraftentfaltung für Senioren eingeführt und zunehmend etabliert. Schnellkrafttraining zeigte größere Effekte auf die Kraftentwicklung und auf funktionelle Fähigkeiten (z. B. „Floor Rise to Stand“-Test, 6-Minuten-Gehtest, „Repeates Chair Rise“- Test) im Vergleich zu traditionellem Krafttraining [25]. Es wirkte sich auch positiv auf die Maximalkraft aus und kann mit einer niedrigeren Gesamtbelastung Transfereffekte zur Balancefähigkeit hervorrufen [8]. Dementsprechend empfiehlt das ACSM für Senioren Schnellkrafttraining mit ein bis drei Sätzen à sechs bis zehn Wiederholungen bei leichter bis moderater Intensität (40-60\% des 1WM) und hohen Bewegungsgeschwindigkeiten [9]. Empfehlungen für Maximalkraftund Schnellkrafttraining nach Granacher et al. [8] sind in - Tab. 1 aufgeführt.

\section{Agility-basiertes Training zur Sturzprävention}

Selbstständig lebende Senioren stürzen aufgrund einer Vielzahl von Ursachen, wie zum Beispiel Stolpern, Ausrutschen, Bewusstseinsverlust, „Frozen Leg“, Verlust einer stabilen Stützfläche, Fehltritte, Schmerzen oder Synkopen [28]. Das Stolpern scheint dabei die häufigste Ursache zu sein [29]. Senioren, die in Pflegeheimen leben, stürzen in mehr als $80 \%$ der Fälle durch falsche Gewichtsverlagerung (41\%), Stolpern, Stoßen oder Verlust einer stabilen Stützfläche (insgesamt 43 \%) [30]. Die Auswirkungen von Kraftund Balancetraining auf das Sturzrisiko und auf Sturzraten sowie das zugrundeliegende Evidenzniveau können als akzeptabel angesehen werden (Level A oder B). Die Rolle der Ausdauerfähigkeit in der Sturzprävention wurde bisher jedoch noch nicht ausreichend untersucht.

Inaktive Senioren, die anfangen, körperlich aktiv zu werden, haben möglicherweise ein erhöhtes relatives Risiko zu stürzen. Außerdem kann die posturale Kontrolle, vor allem 
\ Tab. 1 Übersicht der Empfehlungen für Maximalkraft- und Schnellkrafttraining [8][26]

\begin{tabular}{|c|c|c|}
\hline & Maximalkrafttraining & Explosiv- oder Schnellkrafttraining \\
\hline Volumen & $\begin{array}{l}8-12 \text { Wochen } \\
\text { ca. } 45-60 \text { min pro Einheit } \\
6-8 \text { Übungen pro Einheit } \\
3 \text { Sätze à } 8-12 \text { Wiederholungen }\end{array}$ & $\begin{array}{l}6-12 \text { Wochen } \\
\text { ca. } 45-60 \text { min pro Einheit } \\
6-8 \text { Übungen pro Einheit } \\
3 \text { Sätze à } 8-10 \text { Wiederholungen }\end{array}$ \\
\hline Satzpause & 2 bis 3 Minuten & 1 bis 2 Minuten \\
\hline Frequenz & 2-3 Einheiten pro Woche & 2-3 Einheiten pro Woche \\
\hline Intensität & $\begin{array}{l}70-80 \% \text { des } 1 \text { WM } \\
1 \text { WM: maximal oder submaximal bestimmt (a) } \\
\text { Basierend auf der subjektiv empfundenen Anstrengung } \\
\text { zwischen } 12 \text { und } 16 \text { (Borg-Skala 6-20) }\end{array}$ & $\begin{array}{l}20-70 \% \text { (maximal } 80 \% \text { ) des } 1 \text { WM } \\
70 \% \text { für Explosivkraft } \\
20 \% \text { für Transfereffekte zur Balance } \\
\text { Basierend auf der subjektiv empfundenen } \\
\text { Anstrengung zwischen } 10 \text { und } 13 \text { (Borg-Skala 6-20) }\end{array}$ \\
\hline Geschwindigkeit & $\begin{array}{l}\text { Langsame bis moderate Kontraktionsgeschwindigkeit } \\
\text { während exzentrischer und konzentrischer Arbeit }\end{array}$ & $\begin{array}{l}\text { Schnelle und explosive Kontraktionsgeschwindigkeit } \\
\text { während exzentrischer und konzentrischer Arbeit }\end{array}$ \\
\hline
\end{tabular}

(a) Submaximale 1WM-Schätzungen nach [27], 1WM = Einwiederholungsmaximum

bei Personen mit geringer aerober Kapazität, akut durch aerobe Belastungen im Alltag beeinträchtigt werden: Es gibt Hinweise darauf, dass die Ermüdung nach einem moderaten oder stark belastenden Training (Fahrradfahren oder Gehen) die Balancefähigkeit bei Senioren akut beeinträchtigt [12][31][32]. Andererseits kann möglicherweise eine höhere aerobe Kapazität ermüdungsbedingte Einschränkungen der Balancefähigkeit verringern. Daher sollte auch aerobes Training in Programme zur Sturzprävention integriert werden.

Basierend auf diesen Erkenntnissen erfordert die Sturzprävention ein erfolgreiches Zusammenspiel von Wahrnehmung, kognitiven Fähigkeiten (z. B. Aufmerksamkeit, Planung, Entscheidungsfindung) sowie neuromuskulärer (Rumpf, Beine) und kardiovaskulärer Kapazität. Obwohl aktuelle Empfehlungen zum Balance- und Krafttraining die Aspekte zum Teil zunehmend berücksichtigen (z. B. höhere Bewegungsgeschwindigkeiten und Störeinflüsse im Schnell- und Explosivkrafttraining) [8][33], spiegeln sich die genannten Überlegungen in „Best Practice“-Empfehlungen nicht immer vollständig wider. Neuere Untersuchungen haben sich auf kognitive Aspekte der Entscheidungsfindung unter der Anwendung von Computerspielen und Tablets konzentriert und dabei Elemente funktioneller Mobilität mit Stepping- oder Hindernisaufgaben integriert [34]. Trotz der funktionalen Natur und der Berücksichtigung kognitiver Aspekte dieser Ansätze werden die Kraft- und die Ausdauerkomponente dabei nicht ausreichend einbezogen.

Agility-basierte Trainingsansätze, die eine Kombination von Beschleunigungen, Abbrems- und, Stop-and-GoBewegungen, Richtungsänderungen und exzentrischen Belastungen mit anspruchsvollen räumlichen Orientierungsaufgaben umfassen, können als integratives Trai- ning motorischer Fähigkeiten für Senioren dienen. Solche Agility-basierten Trainingsansätze können Aspekte der Wahrnehmung und Entscheidungsfindung (z. B. visuelle Wahrnehmung, situative räumliche Orientierung, Erkennen und Antizipieren von Bewegungsmustern), Richtungsänderungen (einschließlich reaktiver Bewegungen und schneller konzentrischer sowie exzentrischer Muskelkontraktionen) und kardiovaskuläres Training kombinieren. Ein mehrdimensionales Trainingsprofil auf Grundlage des körperlichen Leistungszustandes des Teilnehmers kann durch eine individuelle Anpassung der Intensität, der Komplexität und der kognitiven Herausforderungen gestaltet werden. Dabei sollten sich physische, perzeptuelle und kognitive Anforderungen im Training zunehmend komplexer und anspruchsvoller gestalten. Auch die kardiovaskulären Belastungen können durch die Auswahl der Übungen, die Anzahl der Übungen pro Zirkel und die Häufigkeit der Ausführung des gesamten Zirkels variieren.

\section{Ein integratives Training bietet eine vielversprechende, ansprechende und zeiteffiziente Trainingsstrategie zur Sturzprävention bei Senioren und kann an die realen Lebensumstände angepasst werden.}

Herkömmliches Balance- und Krafttraining wird häufig als wenig spezifisch für Situationen, in denen das Gleichgewicht bedroht ist, angesehen (z. B. schnelle Reaktion auf plötzliche Störung und schnelles Erzeugen der erforderlichen Gelenkmomente) [8], und Transfereffekte treten kaum auf. Durch den integrativen Ansatz ergeben sich jedoch aufgabenspezifische Anpassungen [35][36], durch die diese Einschränkungen umgangen werden könnten. 
Für eine funktionierende räumliche Orientierung spielen kognitive, physische, vestibuläre und propriozeptive Prozesse eine Rolle, und die Funktion dieser Prozesse nimmt mit dem Alter ab [36]. Durch den beschriebenen Agilitybasierten Ansatz könnten sie in funktionellen und integrativen Bewegungsformen trainiert werden. Die variierenden und komplexen Bewegungsmuster (z. B. Richtungswechsel oder Starts und Stopps) fordern und fördern die vestibuläre

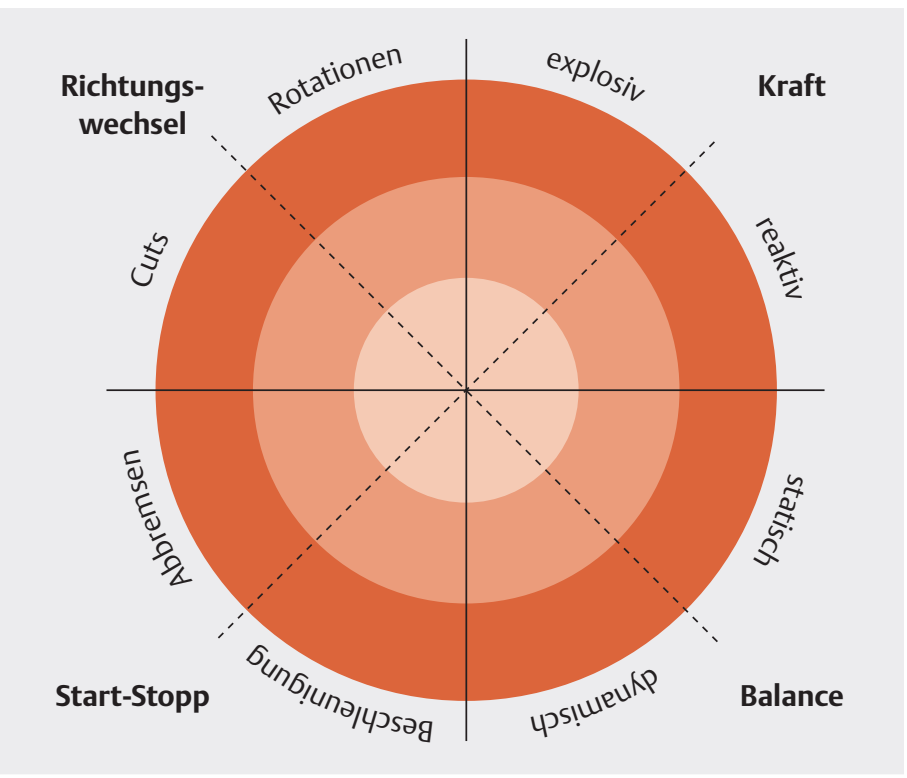

Abb. 2 Modularer Agility-Zirkel mit Richtungswechseln, Starts und Stopps, Kraft- und Balanceanforderungen. Ein Agility-Zirkel kann verschiedene Konditionsformen bei unterschiedlichen Schwierigkeitsgraden (physisch oder kognitiv) akzentuieren, die von hell (hellgrau), mäßig (grau) bis schwer (dunkelgrau) reichen (Quelle: Thieme Gruppe).
Wahrnehmung und stellen erhöhte Anforderungen an die propriozeptive und visuelle Bewegungskontrolle.

Es können beispielsweise verschiedene motorische Komponenten auf unterschiedlichen Schwierigkeitsstufen (physisch und kognitiv) im Vordergrund eines Agility-basierten Trainingszirkels stehen ( $\triangleright$ Abb. 2). Die Schwierigkeit der unterschiedlichen Module ist durch

- die Oberfläche (z. B. fest, Schaum, Matte),

- die Bewegungsgeschwindigkeit,

- die Bewegungsrichtung (z. B. vorwärts, rückwärts, seitwärts),

- die Wiederholungen ( 5 bis 25),

- die Dauer (Anzahl der Module),

- die Sätze (Anzahl der Runden eines Zirkels),

- die Art der Übung (z. B. einfach, komplex, stationär, dynamisch) und

- Störaufgaben (motorisch oder kognitiv)

stufenweise anpassbar.

Ein beispielhafter Agility-Zirkel, der Cutting-Manöver (Richtungswechselmanöver), Rotationsübungen, Beschleunigung und Abbremsen sowie Sprung- und Skipping-Bewegungen umfasst, ist in $\mathbf{A} \mathbf{A b}$. $\mathbf{3}$ dargestellt. Die Auswahl der jeweiligen Module und die zugrunde liegende Schwierigkeit der Übungen können auf Grundlage der individuellen Fähigkeiten und Schwächen getroffen werden.

\section{Schlussfolgerung und Ausblick}

In fortschreitend alternden westlichen Gesellschaften sind Stürze ein ernsthaftes gesundheitliches Problem. Balanceund Krafttraining können Risikofaktoren für Stürze und Sturzraten erfolgreich reduzieren. Aspekte der Ausdau-

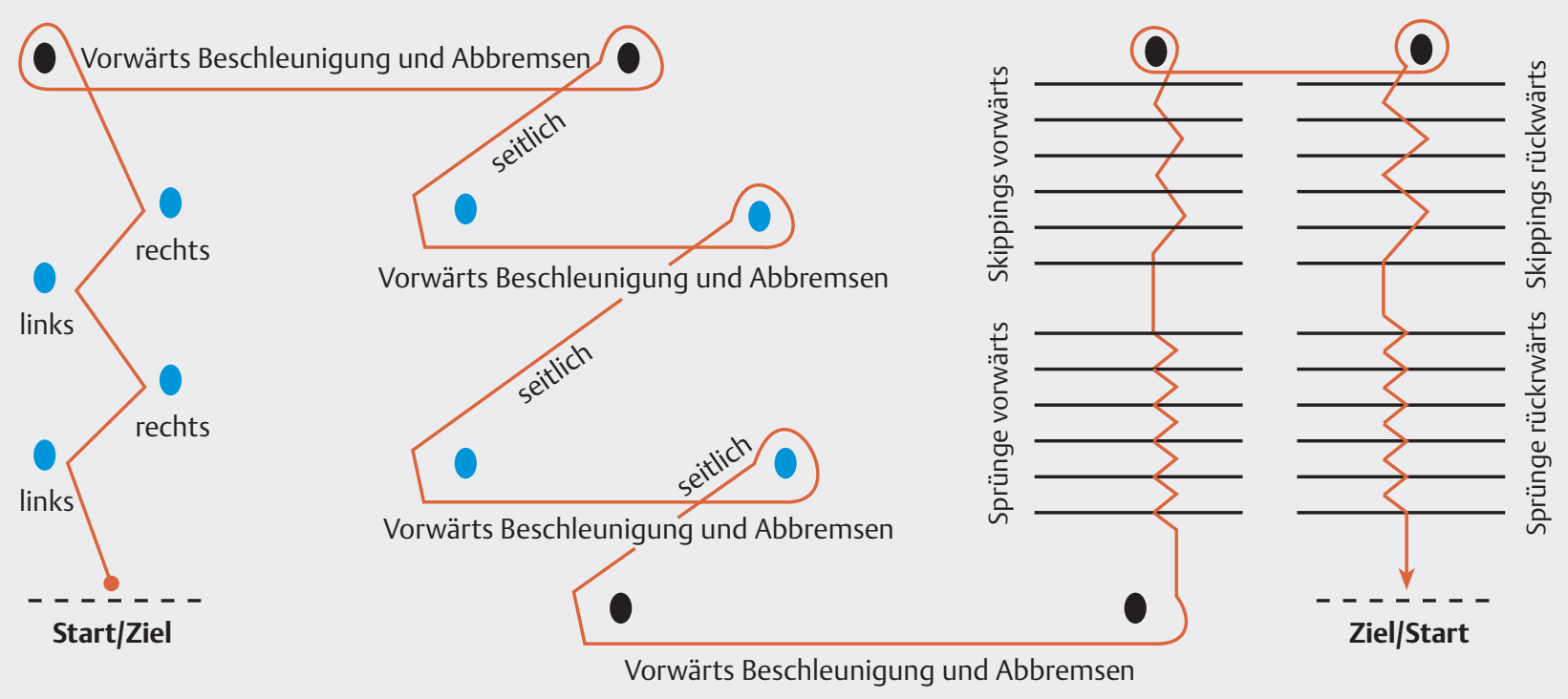

- Abb. 3 Ein Beispiel eines Agility-Zirkels, der Cutting-Manöver, Rotationsbewegungen, Beschleunigung und Abbremsen und Sprung- und Skipping-Bewegungen in verschiedenen Richtungen umfasst (Quelle: Thieme Gruppe). 
er wurden in solchen Trainingsprogrammen bisher kaum berücksichtigt, obwohl eine angemessene aerobe Kapazität nicht nur die kardiovaskuläre Gesundheit verbessert. Sie kann auch zur Sturzprävention beitragen, indem die ermüdungsbedingte Abnahme der posturalen Kontrolle (Haltungskontrolle) während Alltagsbewegungen reduziert wird. In den letzten Jahren standen Schnellkrafttraining, exzentrische Übungen, Gleichgewichtstraining mit Perturbationen sowie Training der Rumpfkraft und -kontrolle im Vordergrund. Ein unabhängiges Training dieser Aspekte wäre einerseits zeitaufwendig und spiegelt andererseits unzureichend das komplexe und funktionelle Anforderungsprofil von sturzrelevanten Situationen wider. Vor diesem Hintergrund erscheint es sinnvoll, Agility-basierte Trainingsformen zu entwickeln sowie in der Praxis zu überprüfen und anzuwenden. Ein solcher funktioneller und integrativer Trainingsansatz könnte durch die Kombination von Muskelkraft, Reaktivität und kognitiven Anforderungen eine Verbesserung der Agilität, der Kraft, der Balance und der Ausdauer hervorrufen. Oberkörper- und Beinmuskeln werden in diesem Konzept in variierenden Bewegungsrichtungen beansprucht. Die Module des Trainings können zusätzlich progressiv angepasst werden, sodass der Zirkel anfangs kurz und leicht und später länger und herausfordernder ist. Dabei können verschiedene neuromuskuläre und kardiovaskuläre Anforderungen gestellt werden, um individuellen Ansprüchen und Fähigkeiten gerecht zu werden. Erste Pilotprojekte unserer Arbeitsgruppe zeigen vielversprechende Ergebnisse und werden zukünftig in großen Interventionsstudien evaluiert.

Der vorgeschlagene konzeptionelle Rahmen sollte weiter bezüglich Validität im Vergleich zu traditionellen Sturzrisikoparametern, Nutzen-Risiko-Verhältnis von möglichen Trainingseffekten in Bezug auf Sicherheitsfragen, psychosozialer Aspekte (Motivation, Freude, Teilnahme, sozialer Interaktion, Adhärenz und Compliance) und Raum- und Materialanforderungen (Sporthalle, Turnhalle erforderlich) untersucht werden.

\section{TAKE HOME MESSAGE}

Agility-basierte Trainingsansätze zur Sturzprävention berücksichtigen Erkenntnisse zahlreicher Untersuchungen zur Sturzprävention. Sie kombinieren unterschiedlichste Übungen mit bewährten Zielsetzungen und können gut dem Gesundheits- und Trainingszustand der Übenden angepasst werden. Kraft und Ausdauer, Gleichgewicht und Wahrnehmung werden in allen relevanten Aspekten trainiert und tragen so wirkungsvoll zu einer Verminderung des Sturzrisikos bei.

\section{Autorinnen/Autoren}

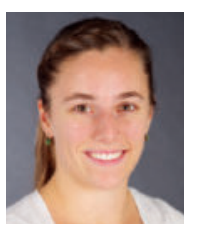

\section{Mareike Morat}

Mareike Morat arbeitet seit 2014 am Institut für Trainingswissenschaft und Sportinformatik der Deutschen Sporthochschule Köln. Hier lagen ihre bisherigen wissenschaftlichen Schwerpunkte in der Leistungsdiagnostik von jungen Kadersportlern und der Erforschung von Anforderungsprofilen verschiedener Berufsgruppen, besonders der Feuerwehr. Im Sommer 2018 schloss sie erfolgreich ihr Masterstudium mit dem Schwerpunk Biomechanik (insbesondere Bewegungsanalyse) an derselben Universität ab. Im Rahmen des Masterstudiengangs absolvierte sie 2015 ein sechsmonatiges Forschungspraktikum im Wolf Orthopaedic Biomechanics Laboratory in London, Kanada. Derzeit befasst sie sich im Rahmen ihrer Promotion mit dem Thema Agility-Training im Alter in der Abteilung für Trainingswissenschaftliche Interventionsforschung der Deutschen Sporthochschule.

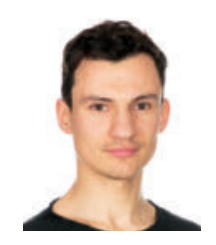

\section{Eric Lichtenstein}

Eric Lichtenstein arbeitet seit 2013 in der Abteilung Bewegungs- und Trainingswissenschaften am Departement für Sport, Bewegung und Gesundheit der Universität Basel. Sein Forschungsschwerpunkt liegt auf der Verbesserung der körperlichen Leistungsfähigkeit beim alternden Menschen und der Verletzungsprävention im Nachwuchsfußball.

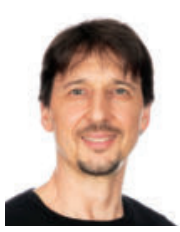

\section{Oliver Faude}

Oliver Faude ist wissenschaftlicher Mitarbeiter und stellvertretender Bereichsleiter im Arbeitsbereich Bewegungs- und Trainingswissenschaft am Departement für Sport, Bewegung und Gesundheit an der Universität Basel. Nach dem Studium der Sportwissenschaften an der Universität des Saarlandes arbeitete er von 2004 bis 2011 als Leiter der ergometrischen Labore in den sportmedizinischen Instituten in Saarbrücken und Paderborn. Dr. Faude zeichnete sich in dieser Zeit u.a. verantwortlich für die leistungsdiagnostischen Untersuchungen aller männlichen Nationalmannschaften des DFB (U16 bis A-Mannschaft). 2011 wechselte er an die Universität Basel. Seine Forschungsschwerpunkte sind Leistungsphysiologie, Verletzungsprävention, Fußballforschung, neuromuskuläre Leistungsfähigkeit sowie die Evaluation und Implementierung von Bewegungsprogrammen in unterschiedlichen Settings und Zielpopulationen.

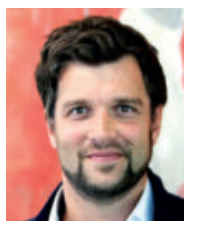

\section{Lars Donath}

Lars Donath (geboren 1980) wurde im Herbst 2017 als Professor für Interventionelle Trainingswissenschaft an die Deutsche Sporthochschule berufen. Zuvor hat er als Doktorand, Habilitand und Vertretungsprofessor an den Universitäten zu Jena (Deutschland), Basel (Schweiz) und Michigan (USA) gearbeitet. Seine Arbeitsschwerpunkte umfassen kardiovaskuläre, -zirkuläre und neuromuskuläre Anpassungsprozesse an Training im Lebensverlauf in klinischen sowie nichtklinischen Populationen. Er ist Autor von mehr als 70 PubMed-gelisteten Artikeln und über fünf Buchprojekten. 
Prof. Dr. Lars Donath

Deutsche Sporthochschule Köln

Institut für Trainingswissenschaft und Sportinformatik

Abteilung Trainingswissenschaftliche Interventionsforschung Am Sportpark Müngersdorf 6

50933 Köln

Tel.: +49 $2214982-7700$

Fax: +49 $2214982-8181$

E-Mail: I.donath@dshs-koeln.de

Literatur

[1] Lutz W, Sanderson W, Scherbov S. The coming acceleration of global population ageing. Nature 2008; 451 (7179): 716-719

[2] Rubenstein LZ. Falls in older people: Epidemiology, risk factors and strategies for prevention. Age and Ageing 2006; 35, Suppl 2: ii37-ii41

[3] Lamb SE, Jørstad-Stein EC, Hauer K et al. Development of a common outcome data set for fall injury prevention trials: The Prevention of Falls Network Europe consensus. Journal of the American Geriatrics Society 2005; 53 (9); 1618-22

[4] Stevens JA, Corso PS, Finkelstein EA. The costs of fatal and non-fatal falls among older adults. Injury Prevention Journal of the International Society for Child and Adolescent Injury Prevention 2006; 12 (5): 290-295

[5] Murray C], Lopez AD. Global mortality, disability, and the contribution of risk factors: Global Burden of Disease Study. Lancet (London, England) 1997; 349 (9063); 1436-42

[6] Sherrington C, Michaleff ZA, Fairhall $N$ et al. Exercise to prevent falls in older adults: An updated systematic review and meta-analysis. British Journal of Sports Medicine 2017; 51 (24): 1750-58

[7] LaStayo PC, Ewy GA, Pierotti DD, . The positive effects of negative work: increased muscle strength and decreased fall risk in a frail elderly population. The Journals of Gerontology. Series A, Biological sciences and medical Sciences 2003: 58 (5); M419-24

[8] Granacher U, Muehlbauer , Zahner L et al. Comparison of traditional and recent approaches in the promotion of balance and strength in older adults. Sports Medicine (Auckland, N.Z.) 2011; 41 (5): 377-400

[9] Chodzko-Zajko W], Proctor DN, Fiatarone Singh MA et al. American College of Sports Medicine position stand. Exercise and physical activity for older adults. Medicine and Science in Sports and Exercise 2009; 41 (7): 1510-30.

[10] Muehlbauer, Roth R, Bopp M et al. An exercise sequence for progression in balance training. Journal of Strength and Conditioning Research 2012; 26 (2): 568-574

[11] Granacher U, Muehlbauer T, Gruber M. A qualitative review of balance and strength performance in healthy older adults: Impact for testing and training. Journal of Aging Research 2012; 2012: 708905

[12] Donath L, Zahner L, Roth R et al. Balance and gait performance after maximal and submaximal endurance exercise in seniors: Is there a higher fall-risk? European Journal of Applied Physiology 2013; 113 (3): 661-669

[13] Skelton DA, Kennedy ], Rutherford OM. Explosive power and asymmetry in leg muscle function in frequent fallers and non-fallers aged over 65. Age and Ageing 2002; 31 (2): 119-125

[14] Gillespie LD, Robertson MC, Gillespie W] et al. Interventions for preventing falls in older people living in the community. The Cochrane Database of Systemic Reviews 2012; (9): CD007146

[15] Karlsson MK, Vonschewelov T, Karlsson C et al. Prevention of falls in the elderly: A review. Scandinavian Journal of Public Health 2013; 41 (5): 442-454

[16] Sherrington C, Tiedemann A, Fairhall $N$ et al. Exercise to prevent falls in older adults: An updated meta-analysis and best practice recommendations. New South Wales Public Health Bulletin 2011; 22 (3-4): 78-83

[17] Donath L, van Dieën J, Faude O. Exercise-based fall prevention in the elderly: What about agility? Sports Medicine (Auckland, N.Z.) 2016; 46 (2): 143-149

[18] Heesch KC, Byles JE, Brown WJ. Prospective association between physical activity and falls in community-dwelling older women. Journal of Epidemiology and Community Health 2008; 62 (5): 421-426

[19] Nelson ME, Rejeski W], Blair SN et al. Physical activity and public health in older adults: Recommendation from the American College of Sports Medicine and the American Heart Association. Circulation 2007; 116 (9): 1094-1105

[20] Tiedemann A, Sherrington C, Close JCT et al. Exercise and Sports Science Australia position statement on exercise and falls prevention in older people. Journal of Science and Medicine in Sport 2011; 14 (6): 489-495

[21] Plummer P, Eskes G. Measuring treatment effects on dualtask performance: A framework for research and clinical practice. Frontiers in Human Neuroscience 2015; 9: 225

[22] Cadore EL, Pinto RS, Bottaro M et al. Strength and endurance training prescription in healthy and frail elderly. Aging and Disease 2014; 5 (3): 183-195

[23] Pijnappels M, van der Burg PJ; Reeves ND et al. Identification of elderly fallers by muscle strength measures. European Journal of Applied Physiology 2008; 102 (5): 585-592

[24] Pijnappels M, Bobbert MF, van Dieën JH. Push-off reactions in recovery after tripping discriminate young subjects, older non-fallers and older fallers. Gait \& Posture 2005; 21 (4): 388-394

[25] Miszko TA, Cress ME, Slade LM et al. Effect of strength and power training on physical function in community-dwelling older adults. The Journals of Gerontology. Series A, Biological Sciences and Medical Sciences 2003: 58 (2): 171-175

[26] Donath L, Faude O, Bopp M et al. Grundlagen gesundheitsorientierten Krafttrainings im Alter: Ziele und Umsetzung. Therapeutische Umschau. Revue therapeutique 2015; 72 (5): $335-342$

[27] Baechle TR, Earle RW (Eds.). Essentials of strength training and conditioning, 3 rd ed. Champaign, IL: Human Kinetics 2008

[28] Zecevic AA, Salmoni AW, Lewko JH et al. Utilization of the Seniors Falls Investigation Methodology to identify systemwide causes of falls in community-dwelling seniors. The Gerontologist 2009; 49 (5): 685-696

[29] Nevitt MC, Cummings SR, Hudes ES. Risk factors for injurious falls: A prospective study. Journal of Gerontology 1991; 46 (5): M164-70

[30] Robinovitch SN, Feldman F, Yang Y et al. Video capture of the circumstances of falls in elderly people residing in longterm care: An observational study. Lancet (London, England) 2013; 381 (9860): 47-54 
[31] Stemplewski R, Maciaszek], Salamon A et al. Effect of moderate physical exercise on postural control among $65-74$ years old men. Archives of Gerontology and geriatrics 2012; 54 (3): e279-83

[32] Donath L, Kurz E, Roth R et al: Does a single session of highintensity interval training provoke a transient elevated risk of falling in seniors and adults? Gerontology 2015; 61 (1): $15-23$

[33] Granacher U, Gollhofer A, Hortobágyi T et al. The importance of trunk muscle strength for balance, functional performance, and fall prevention in seniors: A systematic review. Sports Medicine (Auckland, N.Z.) 2013; 43 (7) 627-641

[34] Granacher U, Bridenbaugh SA, Muehlbauer T et al. Age-related effects on postural control under multi-task conditions. Gerontology 2011; 57 (3): 247-255
[35] Donath L, Roth R, Rueegge A et al. Effects of slackline training on balance, jump performance $\&$ muscle activity in young children. International Journal of Sports Medicine 2013; 34 (12): 1093-98

[36] Lackner JR, DiZio P. Vestibular, proprioceptive, and haptic contributions to spatial orientation. Annual review of Psychology 2005; 56: 115-147

Bibliografie

DOI https://doi.org/10.1055/a-0818-3107

Sportphysio 2019; 7: 22-29

(c) Georg Thieme Verlag KG Stuttgart · New York ISSN 2196-5951 